Ann. Zootech., I972, 21 (I), II3-I20.

NOTE

\title{
OBSERVATIONS PRÉLIMINAIRES \\ SUR LES VARIATIONS SAISONNIÈRES \\ DE LA QUANTITÉ D'ALIMENT INGÉRÉE \\ PAR LES GAPRINS EN MILIEU TROPICAL HUMIDE
}

\author{
M. CHENOST \\ avec la collaboration technique de P. Despors et A. Grude \\ Station de Recherches zootechniques, \\ Centre de Recherches agronomiques des Antilles et de la Guyane, I. N.R.A., \\ Petit-Bourg, Guadeloupe
}

Dans le cadre de l'intensification fourragère en zone tropicale humide, le problème de la connaissance de la valeur alimentaire (digestibilité et quantité ingérée) des graminées à haut potentiel de production et celui de leur utilisation par des animaux de race améliorée, importée de zones tempérées, devient extrêmement important. De nombreuses études ont été réalisées dans ce sens en zone Caraïbe (Osbourn, 1969 ; Butterworth, I965; Grieve et Osbourn, r965 ; Rivera-Brenes et al., 1961; Vincente-Chandeer et al., i964; Creer, 197o, etc.). La Station de Recherches zootechniques du C. R. A. A. G. en Guadeloupe a, quant à elle, abordé cette étude avec un petit ruminant, la chèvre laitière, qui a été peu utilisée jusqu'ici en zone tropicale humide.

Nous avons poursuivi depuis I968 l'étude systématique de la digestibilité des fourrages tropicaux et de la quantité qui en est ingérée par les caprins qui les reçoivent à volonté soit seuls, soit avec un complément d'aliment concentré. Nous ne rapportons ici que les résultats des mesures de digestibilité et de quantités ingérées effectuées pendant deux ans sur des boucs qui recevaient à l'auge des repousses de Pangola (Digitaria decumbens) présentées seules. Nous nous sommes attachés à mettre en évidence l'influence de la saison sur les quantités ingérées ; celle-ci peut être, en effet, très importante d'après les travaux de Worstell et Brody (I953), (Findlay, 1954), Payne et Hancock (1957). Johnson et Ragsdale (I960), Sharma, i968).

\section{MATERIEL ET METHODES}

\section{Principe}

Sous le climat tropical humide de Guadeloupe $(2,9 \mathrm{~m}$ d'eau par an répartis à peu près uniformément sur l'année sauf pendant les mois de janvier à mars), le Pangola présente une période de croissance très marquée allant de mai à octobre pendant laquelle il réépie à chaque repousse. 
De novembre à avril sa croissance est ralentie et il se présente sous forme de repousses feuillues.

Nous avons mesuré l'évolution tout au long de l'année de la digestibilité et des quantités ingérées de repousses de Pangola :

- d'une part "d'âge constant " provenant de deux prairies exploitées en continu, subdivisées l'une en 30 parcelles ( 30 jours), l'autre en $5^{\circ}$ parcelles (5o jours), une parcelle correspondant à la surface fauchée chaque jour ;

- d'autre part " d'âge variable " allant de 28 à 77 jours provenant d'une prairie exploitée à partir de 28 et jusqu'à 77 jours après la fauche d'égalisation précédente.

La prairie exploitée à 30 jours d'âge recevait $84^{\circ} \mathrm{kg}$ d'azote et $84^{\circ} \mathrm{kg}$ de potasse par hectare et par an, celle exploitée à 50 jours d'âge recevait $350 \mathrm{~kg}$ d'azote et $350 \mathrm{~kg}$ de potasse par hectare et par an; la fumure phosphorique était respectivement égale à 250 et $130 \mathrm{~kg}$.

La prairie exploitée par cycles successifs d'âge variable recevait une fumure de $80 \mathrm{~kg}$ d'azote et de $80 \mathrm{~kg}$ de potasse à chaque fauche d'égalisation, l'acide phosphorique étant apporté une fois par an $\left(25^{\circ} \mathrm{kg}\right)$. Ce mode d'exploitation avait été choisi dans le but de récolter des fourrages de caractéristiques et de valeur nutritive différentes.

Les fourrages ont été distribués à 3 lots de 6 boucs $(4$ en cage à digestibilité, 2 en case au sol). Un lot a reçu les repousses d'âge variable ( 13 cycles d'exploitation successifs pendant deux ans) ; les deux autres lots ont reçu en permanence pendant un an et demi les repousses d'âge constant.

Le fourrage frais, fauché le matin entre 7 et $9 \mathrm{~h}$, était distribué à volonté (Io p. Ioo de refus) à l'auge en deux repas, l'un au début de la matinée, l'autre dans le milieu de l'après-midi.

\section{Animaux}

Les boucs utilisés étaient de race alpine (Chamoisée et Saanen). Ils avaient été importés de France en I966 ou étaient nés en Guadeloupe. Entiers pour la plupart, ils étaient âgés de 2 à 4 ans et pesaient entre 37 et $65 \mathrm{~kg}(49,5 \mathrm{~kg}$ en moyenne).

\section{Mesures}

Les quantités de matière sèche ingérées ont été mesurées chaque jour, sauf le dimanche, par pesée des quantités offertes et refusées et détermination de la teneur en matière sèche du fourrage distribué et des refus. Chaque résultat correspond à la moyenne des consommations journalières individuelles de 6 boucs pendant la période du lundi au samedi.

La digestibilité de chaque catégorie de fourrage vert a été mesurée avec les quatre boucs en cage ; chaque résultat correspond à la digestibilité moyenne du fourrage offert pendant la période du lundi au samedi.

Un échantilion représentatif du fourrage offert et refusé a été constitué pour chaque période de mesure; nous en avons fait l'analyse morphologique et l'analyse chimique (teneur en cendres, en azote et cellulose brute Weende).

Les températures moyennes mensuelles, minimales, maximales et moyennes, ont été relevées par la Station de bioclimatologie du C. R. A. A. G.

\section{RESULTATS}

\section{Repousses d'age constant}

Nous avons rapporté, sur la figure I, les variations saisonnières de la teneur en matière sèche, en matières azotées et en feuilles des deux repousses d'âge constant.

- Les teneurs en matière sèche des repousses de $5^{\circ}$ jours ont toujours été d'environ 6 points plus élevées que celles des repousses de 30 jours; elles n'ont pas varié de façon marquée et régulière suivant la saison.

- La teneur en matières azotées digestibles des repousses de 30 jours, d'une valeur moyenne de 10,8 p. Ioo, a varié entre 5 , I et I $_{5,2}$ p. Ioo; celle des repousses de 50 jours, d'une valeur moyenne de 5,9 p. ıоo, a varié entre 3,2 et ıo,2 p. ıоo. Dans les deux cas, elle présente des varia- 
tions saisonnières marquées ; elle est minimum entre mai et septembre, période de forte croissance pendant laquelle le Pangola réépie et a le pourcentage de feuilles le plus faible (fig. I); elle est d'ailleurs en liaison avec ce dernier $(\gamma=0,746, n=4 \mathrm{I})$.
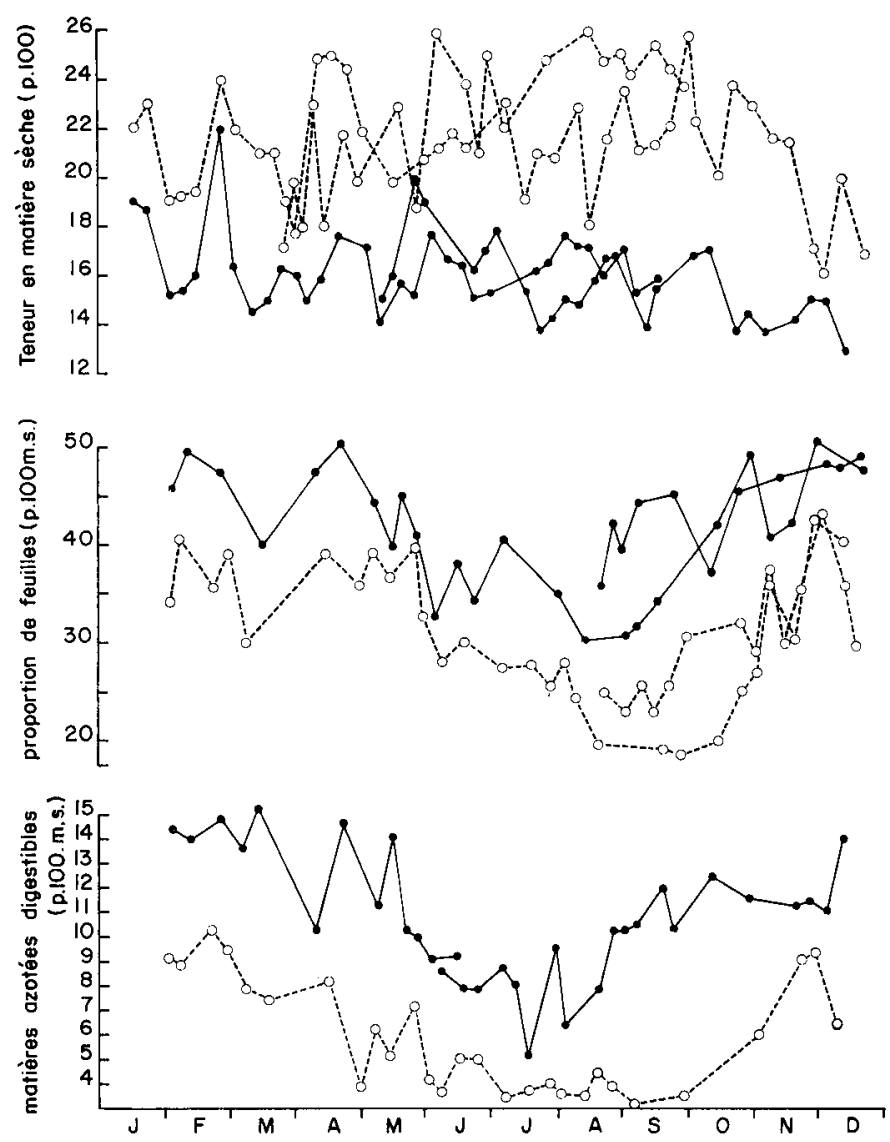

FIG. I. - Variation au cours de l'année de la teneur en matières azotées digestibles, de la proportion de feuilles et de la teneur en matière sèche de deux repousses de Pangola (Digitaria decumbens) d'age constant, l'une de $30 j(\bullet-\bullet)$, l'autre de $50 \mathrm{j}(0-\ldots . \cdots)$.

Nous avons rapporté, sur la figure 2, les variations saisonnières de la digestibilité et des quantités ingérées.

- La digestibilité de la matière organique des repousses de 30 jours a été comprise entre 62 et 74 p. Ioo $(68,5$ p. Ioo en moyenne), celle des repousses de 50 jours entre 55 et 65 p. 100 $(60,5$ p. roo en moyenne). Différentes en moyenne de 8 points elles ont toutes deux eu tendance à diminuer légèrement pendant les mois de juillet à septembre.

- Les quantités de matière sèche ingérées ont varié dans des limites très larges ( $\mathrm{I}, 00$ à 2,60 kg/100 kg de poids vif); elles ont parfois été plus élevées avec les repousses de 50 jours qu'avec les repousses de 30 jours (février à mars). L'ingestion maximale a eu lieu pendant les mois de février, mars et avril où elle a dépassé $2,00 \mathrm{~kg}$ et atteint plus de $2,50 \mathrm{~kg} / \mathrm{roo} \mathrm{kg}$ de poids vif; elle a ensuite diminué à partir du mois de mai pour atteindre le minimum pendant les mois de juillet à décembre, pendant lesquels elle a, d'ailleurs, été relativement variable $(1,00$ à $2,00 \mathrm{~kg} /$ roo $\mathrm{kg}$ de poids vif) (fig. 2). 
- Les quantités de matière organique digestible ingérées ont varié de 18 à $4^{\circ} \mathrm{g}$ par $\mathrm{kg}$ de poids métabolique, elles n'ont pas été significativement différentes suivant l'âge des repousses et ont présenté les mêmes variations saisonnières que les quantités de matière sèche ingérées (fig. 2).
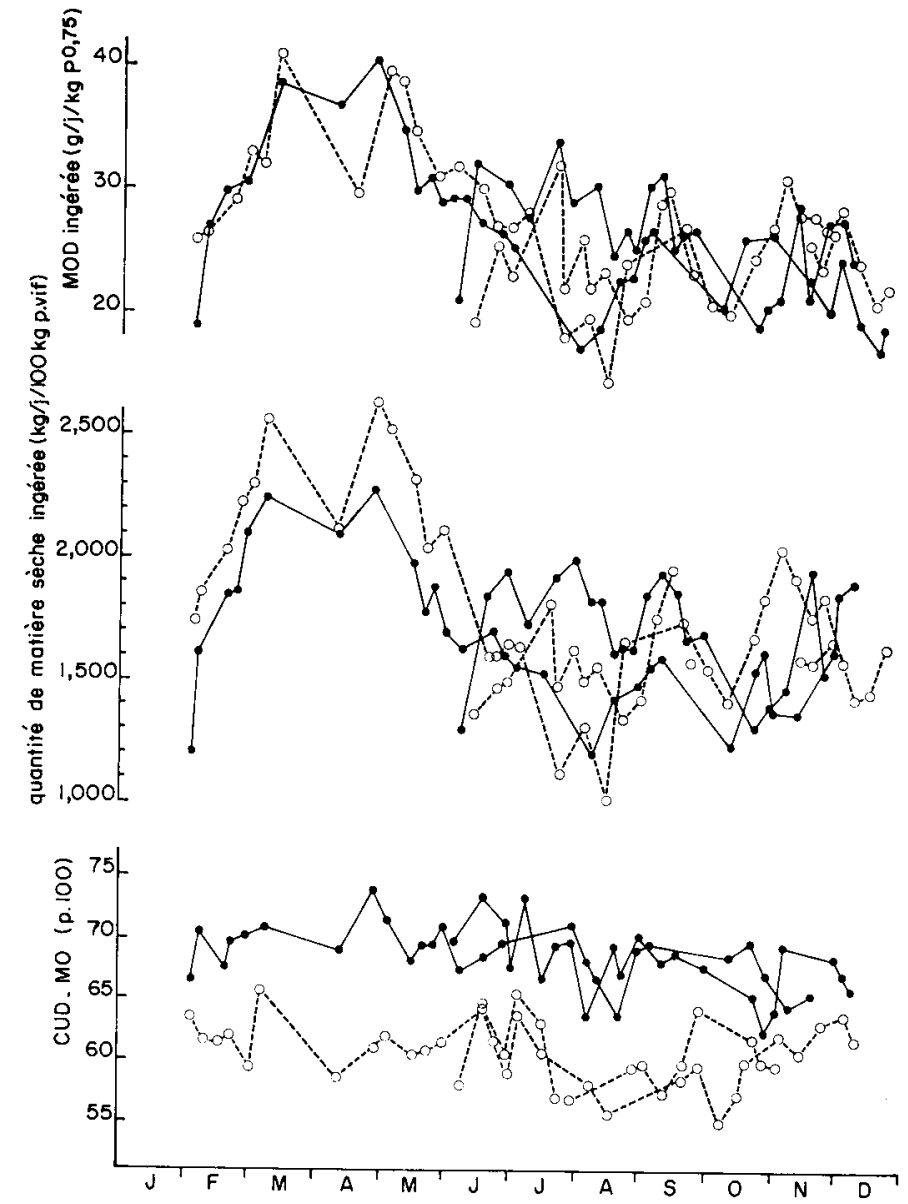

FIG. 2. - Variation au cours de l'année de la digestibilitê de la matière organique, des quantités de matière sèche et de matière organique digestible ingérées de deux repousses de Pangola d'áge constant l'une de $30 j(\bullet-\bullet)$, l'autre de $50 j(0-\ldots .-0)$.

Nous n'avons pas observé, pour l'ensemble des deux catégories de repousses, de liaison significative entre les quantités de matière sèche ingérées et, soit la teneur en matière sèche, soit la digestibilité. Le pourcentage de feuilles a présenté une liaison d'ailleurs assez lâche $\left(r=0,43^{\circ}, n=69\right)$ avec la quantité ingérée, cette liaison devenant plus étroite lorsqu'on ne considère que les repousses d'avril à octobre $(r=0,521, n=33)$; il en a été de même de la teneur en matières azotées digestibles qui est, d'ailleurs, étroitement liée au pourcentage de feuilles $\left(r=0,75^{6}\right)$.

\section{Repousses d'âge variable}

Nous n'avons rapporté ici (fig. 3) que les variations saisonnières des quantités de matière sèche ingérées. La digestibilité de la matière organique diminue en effet relativement peu avec 


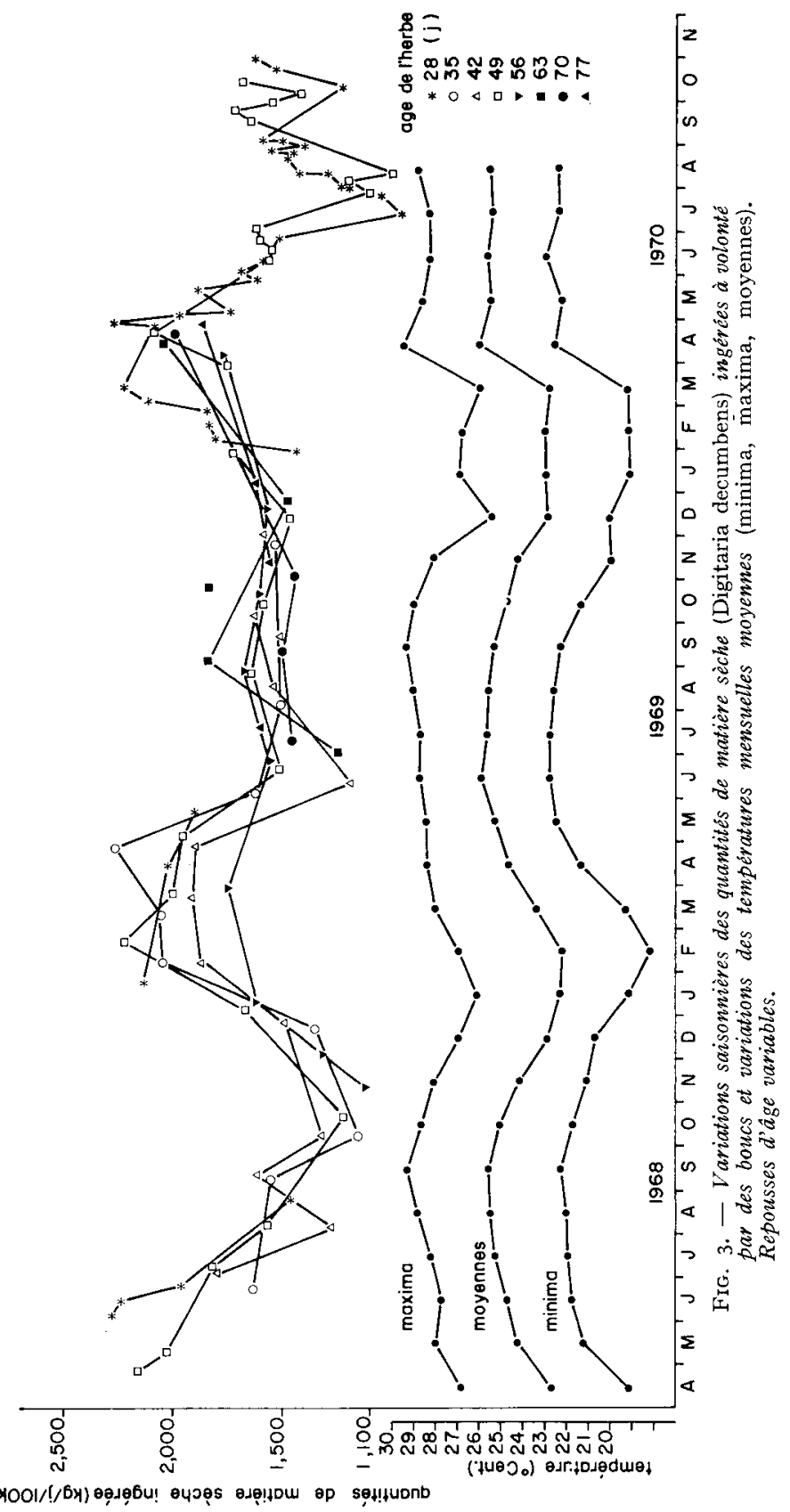


l'âge (de 70,5 p. Ioo à 28 jours à 60,0 p. I00 à 77 jours) et ne présente pas, à un âge donné, de variations saisonnières importantes.

Les quantités de matière sèche ingérées ont varié de 0,98 à $2,29 \mathrm{~kg}$ par Ioo $\mathrm{kg}$ de poids vif suivant l'âge de la plante et la saison (fig. 3). Elles ont été en liaison très lâche avec la digestibilité $(r=0,3$ Io, $\mathbf{P}<0$,o5) et avec la teneur en matières azotées digestibles $(r=0,333$, $\mathbf{P}<0,05)$.

Les variations suivant la saison ont été reproductibles. Elles ont présenté un maximum pendant les mois de février, mars et avril et un minimum pendant les mois de juillet à décembre (fig. 3). L'amplitude de ces variations a été plus importante en I968 qu'en r969, et a été voisine de I $\mathrm{kg}$ par Ioo $\mathrm{kg}$ de poids vif. C'est au moment où les températures ont présenté leur minimum que les quantités ont commencé à augmenter (fig. 3).

L'amplitude des variations des quantités ingérées due à l'âge des repousses a toujours été inférieure à celle due à la saison. Elle a été maximum (0,5 kg par ıoo $\mathrm{kg}$ de poids vif) en mars I 969 et en avril 1970, les fourrages ayant alors un âge variant respectivement de 35 à 56 jours et de 28 à 56 jours ; elle a été minimum $(0,2 \mathrm{~kg}$ par $100 \mathrm{~kg}$ de poids vif) pendant les mois de juillet à décembre.

\section{DISCUSSION}

Nous constatons que les quantités ingérées présentent des variations saisonnières importantes et reproductibles. Quelles sont les causes de ces variations saisonnières ? La saison agit-elle directement sur l'appétit des animaux ou sur les caractéristiques de l'acceptabilité des fourrages?

En effet, la température et 1'hygrométrie pourraient, en modifiant la régulation thermique de l'animal, agir sur l'appétit (Worstell et Brody, I953; Findlay, 1954, cités par Payne en I966; PAYNe et HANCOCK, I957 ; Sharma, I968). Il semble, d'ailleurs, d'après nos résultats, que les variations de température ambiante aient joué un rôle sur les quantités ingérées (fig. 3). Il ne nous a malheureusement pas été possible de mettre en parallèle avec les quantités ingérées, l'hygrométrie qui doit cependant être un élément non négligeable de la régulation thermique de l'animal et de son bien-être (Johnson et Ragsdale, ig60, cité par Payne, ig66). Il est cependant possible que l'influence de la saison sur l'appétit ait été amplifiée chez les boucs par le regain d'activité sexuelle que les animaux non castrés ont manifesté de juillet à octobre, c'est-à-dire durant la période la plus chaude et humide.

La saison pourrait aussi agir sur les quantités ingérées en modifiant l'ingestibilité des fourrages : teneur en matière sèche, composition chimique et morphologique (notamment teneur en azote, en membranes, en feuilles et en parties mortes) et aptitude du fourrage à être dégradé plus ou moins rapidement dans le rumen. C'est ainsi, d'ailleurs, que Deinum et al., (ı968) et Minson et al., (I970), pensent que la température pourrait avoir une action directe sur la digestibilité des fourrages.

D'après nos résultats obtenus sur les repousses d'âge constant il ne semble pas que les modifications des teneurs en matière sèche, en azote et en feuilles, suivant la saison, aient été responsables de variations aussi importantes des quantités ingérées. En effet, malgré des teneurs en matières azotées et en feuilles, et des digestibilités aussi élevées en octobre, novembre et décembre qu'en février, mars et avril, les quantités de matière sèche et de matière organique digestible ingérées ont été bien plus importantes pendant les mois de février à avril que pendant ceux d'octobre à décembre.

Il nous reste, toutefois, à préciser quelle est la part revenant à chacun de ces deux modes d'action.

Dans l'immédiat, ces résultats ont deux intérêts. Le premier est d'ordre méthodologique. Nous ne pouvons comparer la valeur alimentaire (digestibilité et quantités ingérées) des différentes espèces ou variétés de fourrages tropicaux que si les mesures ont lieu simultanément, ou, tout au moins, si clles sont très rapprochées dans le temps. Si les périodes de croissance des 
fourrages à comparer sont différentes, il sera nécessaire d'étudier ces fourrages après congélation ou déshydratation, ces deux techniques de conservation modifiant peu la valeur alimentaire des fourrages et notamment des quantités ingérées (RAymond et al., 1953; Demarquilly et JARRIGE, I970). Le deuxième intérêt est d'ordre zootechnique. Il peut être avantageux de faire coïncider les débuts de lactation des chèvres avec des époques où l'ingestion des fourrages est maximum et cela d'autant plus que les chèvres seront en fin de lactation, donc auront des besoins minimaux, à l'époque où les quantités de fourrages ingérées sont les plus faibles.

Reçu pour publication en aồt $19 \% 1$.

\section{SUMMARY}

\section{SEASONAL, VARIATIONS OF THE VOLUNTARY FOOD INTAKE BY GOATS IN HUMID TROPICAI, CLIMATE. PRELIMINARY STUDY.}

The variations in dry matter intake and digestibility of Pangola grass (Digitaria decumbens) regrowths of constant age ( 30 and 50 days) were studied during one and a half years and those of Pangola regrowths of variable age (28-77 days), during two years.

The measurements were made on male goats of the Alpine breed offered green forage, ad libitum, twice a day (morning and afternoon). The contents of dry matter and crude protein and the proportion of leaves in the regrowths of constant age were determined.

I. The crude protein content (mean values : I0.8 and 5.9 per cent for regrowths of 30 and 50 days respectively) was at a maximum from May to September (fig. I) i.e. period of maximum growth of Pangola grass with formation of ears at each harvest. The dry matter content of the forage showed small seasonal variations (fig. I).

The proportion of leaves showed the same seasonal variations as the crude protein content.

The mean digestibility of the organic matter was 68.5 and 60.5 per cent for regrowths of 30 and 50 days respectively and showed only slight seasonal variations (fig. 2).

The dry matter intake varied within very wide limites (I.oo to $2.60 \mathrm{~kg}$ DM per $100 \mathrm{~kg}$ live weight), but was not significantly different according to the age of the regrowths and was at a maximum from February to April (fig. 2). The dry matter intake was not related to the dry matter content and digestibility, and only slightly related to the proportion of leaves

$$
(r=0.430 n=69)
$$
(fig. 2).

The digestible organic matter intake varied in the same manner as the dry matter intake

2. The dry matter intake from the regrowths of different ages also showed important seasonal variations (fig. 3) which were repeatable. The magnitude of the variations in intake due to season was twice that due to age of the forage offered (I.o and $0.5 \mathrm{~kg}$ dry matter $/ \mathrm{kg}$ live weight for season and age respectively).

The seasonal variations of the voluntary food intake appear to depend more on variations of the climatic conditions (temperature-humidity) than on variations of the specific characteristics of the forage since the food intake between the seasons, February-April and OctoberDecember, was very different whereas the digestibility, crude protein content and proportion of leaves were similar.

\section{RÉFÉRENCES BIBLIOGRAPHIQUES}

Butterworth M. H., Butterworth, Juanita P., I965. Some aspects of the utilization of tropical forages. 2. Pangola and Coastal Bermuda hays. J. agric. Sci., 65, 389-395.

Creek M. J., I970. Intensification of pasture production with beef breeding herds maintained upon improved pasture (Digitaria decumbens) in Jamaïca. Proc. 11th Internat. Grassl. Congr., 80o-803.

Deinum B., Van Es A. J. H., Van Soest J. P., ig68. Climate, nitrogen and grass. II. The influence of light intensity, temperature and nitrogen on in vivo digestibility of grass and the prediction on these effects from chemical procedures. Neth. J. Agric. Sci, 16, 217-223. 
Demarquilly C., JARrige R., I970. The effect of method of forage conservation on digestibility and voluntary intake. Proc. 11th Internat. Grassl. Congr., 733-737.

Grieve C. M., Osbourn D. F., I965. The nutritional value of some tropical grasses. J. Agric. Sci., 63, $253-259$.

Minson D. J., McLeod N. M., I97o. The digestibility of temperate and tropical grasses. Proc. 11 th Internat. Grassl. Congr., 7I9-722.

Osbourn D. F., I969. The introduction of Pangola grass in the Caribbean islands. J. Brit. Grassl. Soc., 24, 76-80.

Payne W. J. A., I966. Nutrition of ruminants in the tropics. Nutr. abstracts and reviezes, 36, 653-67o.

Raymond W. F., Harris C. E., Harker, V. G., 1953. Studies on the digestibility of herbage. II. Effect of freezing and cold storage of herbage on its digestibility tests by sheep. J. Brit. Grassl. Soc., 8, 3I5-320.

Rivera-Brenes L. et al., I96I. Palability tests on geant Pangola (Digitaria valida stent) and signal grass (Brachiaria brizantha) as compared with Napier (Merker) grass (Pennisetum purpureum). J. A gric. Of the University of Puerto-Rico, 45, I47-I5o.

Sharma D. C., r968. Intake and digestion of nutrients by the bovines under climatic stress. J. Nutr. 94, 317-325.

Vincente-Chandler J. R. et al., I964. The intensive management of tropical forages in Puerto-Rico Bull. 187 , Univ. of Puerto-Rico, 5-I52. 